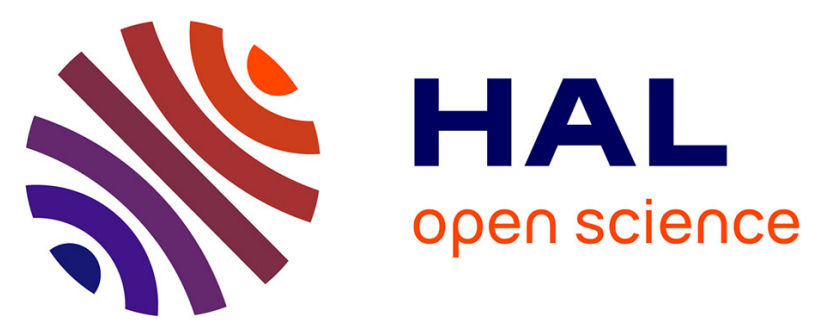

\title{
Scintillation properties of N2 and CF4 and performances of a scintillating ionization chamber
}

G. Lehaut, S. Samuel Salvador, J.M. Fontbonne, F.R. Lecolley, J. Perronnel, Ch. Vandamme

\section{- To cite this version:}

G. Lehaut, S. Samuel Salvador, J.M. Fontbonne, F.R. Lecolley, J. Perronnel, et al.. Scintillation properties of $\mathrm{N} 2$ and $\mathrm{CF} 4$ and performances of a scintillating ionization chamber. Nuclear Instruments and Methods in Physics Research Section A: Accelerators, Spectrometers, Detectors and Associated Equipment, 2015, 797, pp.57-63. 10.1016/j.nima.2015.05.050 . in2p3-01170130

\section{HAL Id: in2p3-01170130 \\ https://hal.in2p3.fr/in2p3-01170130}

Submitted on 3 Jul 2015

HAL is a multi-disciplinary open access archive for the deposit and dissemination of scientific research documents, whether they are published or not. The documents may come from teaching and research institutions in France or abroad, or from public or private research centers.
L'archive ouverte pluridisciplinaire HAL, est destinée au dépôt et à la diffusion de documents scientifiques de niveau recherche, publiés ou non, émanant des établissements d'enseignement et de recherche français ou étrangers, des laboratoires publics ou privés.

\section{(ㅇ)(1) $\$$}

Distributed under a Creative Commons Attribution - NonCommercial| 4.0 International 


\title{
Scintillation properties of $\mathrm{N}_{2}$ and $\mathrm{CF}_{4}$ and performances of a scintillating ionisation chamber
}

\author{
G. Lehaut, S. Salvador, J.-M. Fontbonne, F.-R. Lecolley, J. Perronnel and Ch. Vandamme. ${ }^{1}$ \\ ${ }^{1}$ LPC Caen, ENSICAEN, Université de Caen, CNRS/IN2P3, Caen, France
}

\begin{abstract}
In this work, we studied the emission yields, decay times and coincidence resolving times (CRT) of two gases, nitrogen $\left(\mathrm{N}_{2}\right)$ and tetrafluoromethane $\left(\mathrm{CF}_{4}\right)$, used for particle detection in the context of fission products measurement. The set-up was made of an ionization chamber and two photomultiplier tubes (PMTs) placed front-to-front on each side of the active zone of the chamber. Using the photomultiplier tubes, the number of photoelectrons (phe) converted at the photocathodes from the scintillation processes in each gas were quantified and the scintillation time spectra were recorded. An scintillation emission yield of 24 phe $\mathrm{MeV}^{-1}$ with a decay time of $\tau_{d}=2.5 \mathrm{~ns}$ in $\mathrm{N}_{2}$, and 225 phe $\mathrm{MeV}^{-1}$ with $\tau_{d}=6.2 \mathrm{~ns}$ for $\mathrm{CF}_{4}$, have been measured. With our set-up, the coincidence resolving time ( $\sigma$ values) between the two PMTs have been measured at $1.4 \mathrm{~ns}$ and $0.34 \mathrm{~ns}$ for $\mathrm{N}_{2}$ and $\mathrm{CF}_{4}$ respectively, using alpha particles.
\end{abstract}

\section{INTRODUCTION}

Ionisation chambers (ICs) have been widely used in many fields of nuclear physics due to their good energy resolution, large acceptance and low production cost. Currently, the SPIDER [1] and FALSTAFF [2] projects are devoted to fission studies and intend to use ionization chambers together with two additional Secondary Electron Detectors (SED) in order to measure the energy and the velocity of one fission fragment. They thereby both rely on the determination of the fragment Timeof-Flight (ToF) between SED foils, made of carbon coupled to Micro-Channel Plates (MCP) for SPIDER and mylar associated with micromegas gaseous chambers for FALSTAFF. The energy of the fragment being measured by the IC. In those projects, high energy and time resolutions are required to obtain a good mass identification resolution. For instance, the framework of the FALSTAFF project at the NFS[29] facility, requires a $1 \%$ energy resolution and a 150 ps time resolution FWHM to be able to measure fission fragments masses with a 2 units resolution [2]. However, if ICs can ensure a 1\% energy resolution, difficulties arise in obtaining a good event energy reconstruction through the lack of knowledge of the energy losses encountered by the fission fragments in the two SED foils and the IC entrance window.

To improve the reconstruction performances of the detection system, we have studied the possibility to replace one SED by the fast triggering signal obtained from the scintillation processes induced in the gas of the IC. This system could find applications in neutron beam energy monitoring by ToF like systems [3] for instance in the case of neutron cross sections measurements where the ionisation chamber is used as an active target [4].

Many works reported the good transport properties of electrons in tetrafluoromethane $\left(\mathrm{CF}_{4}\right)$ compiled in [5]. $\mathrm{CF}_{4}$ scintillation properties [6-10], and those of nitrogen $\left(\mathrm{N}_{2}\right)[3,11]$, are already well established. These two gases were thereby investigated as good candidates for use in the scintillating ionisation chamber (SIC). In this paper, we propose to study their scintillation properties relevant for use in our IC (i.e. emission yields and decay times) as well as the energy and timing performances of the resulting SIC. Those measurements ought to be integrated in a Monte-Carlo simulation for the detection system oncoming evaluations.

\section{MATERIALS AND METHODS}

\section{Experimental set-up}

Fig. 1 shows the apparatus used to study the primary scintillation yields and decay times of $\mathrm{N}_{2}$ and $\mathrm{CF}_{4}$. In the gas vessel, the active volume of $5 \times 5 \times 5 \mathrm{~cm}^{3}$ of the ionization chamber was delimited by field shaping wires, a cathode and a Frish Grid (FG). The high voltage of $1182 \mathrm{~V}$ applied between the cathode and the FG made a $236 \mathrm{~V} \mathrm{~cm}^{-1}$ electric field in the active volume. The field shaping wires were used to obtain a constant electric field from the cathode to the FG, ensuring a constant drift velocity of the charges. The field between the grounded anode and the FG with a distance of $0.35 \mathrm{~cm}$ was set to $337 \mathrm{~V} \mathrm{~cm}^{-1}$ in order to increase the transparency up to $95 \%$ of the incoming electrons from the active volume. This value was obtained by Monte Carlo simulation based on electric field maps calculated using a Finite Element Method software (FreeFEM++ [12]). The inefficiency $(\sim 4 \%)$ of the grid was estimated by calculations as in [13].

The radioactive source used for the measurements was placed beneath the cathode inside the ionizing volume in order to reduce energy losses. The source was made by electro-deposition on a stainless steel disc of three radioactive elements, ${ }^{239} \mathrm{Pu},{ }^{241} \mathrm{Am}$ and ${ }^{244} \mathrm{Cm}$, emitting $\alpha$ particles with mean energies of $E_{\alpha}=5.14,5.44,5.8 \mathrm{MeV}$ respectively, in $2 \pi$ sr within the active volume.

The two photomultiplier tubes, R-2059 from Hamamatsu corp., were located front-to-front on both side of 


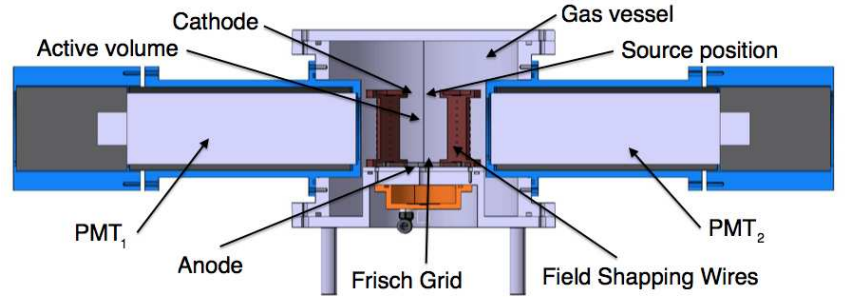

FIG. 1: Sketch of the experimental set-up.

the active volume. The wavelength at maximum emission of $\mathrm{N}_{2}$ was evaluated at $390 \mathrm{~nm}$ [14]. According to [8], $\mathrm{CF}_{4}$ exhibits two main components at $\sim 300 \mathrm{~nm}$ and $\sim 630 \mathrm{~nm}$. The scintillation emission spectra of the gases matches then the PMTs spectral response which ranges from 160 to $650 \mathrm{~nm}$ from the manufacturer data sheets, with a peaked sensitivity measured at $320 \mathrm{~nm}$ [15].

The PMTs entrance windows were used as sealing interfaces with the gas while leakages were avoided using torus joints. The high voltages were set to $-2200 \mathrm{~V}$ corresponding to an intrinsic gain of approx. $10^{6}$.

The gases, nitrogen (from Air Liquide) and tetrafluoromethane (provided by Messer UHP), were injected in the active volume at a $1 \mathrm{~atm}$ pressure. The detector operates in gas flow with a debit of $\sim 0.25 \mathrm{~L} \mathrm{~h}^{-1}$.

The electric signals from the PMTs were converted into charge integrating channels (QDCs) using the FASTER in-house built acquisition system [16]. Each acquisition input was also able to measure the time of arrival of the signals using a $7.8 \mathrm{ps}$ time-to-digital converter (TDC) fed by a digital constant fraction discriminator (dCFD). The signal coming from the IC passed through a charge pre-amplifier with a $\tau_{R C}$ of $30 \mu \mathrm{s}$ and was acquired using a third input used as an amplitude-to-digital converter (ADC) with a 2 ns time stamp. Each input was recorded individually and the analysis was performed off-line. The signal processing were chosen to optimize the timing resolutions for the PMTs, especially on the dCFD, as well as for the energy resolution of the IC.

\section{IC Energy resolution}

The IC detected the signals induced by secondary electrons resulting of the interactions of $\alpha$ particles with the gas molecules. The signal was processed by a numerical algorithm that mimics a spectroscopic amplifier. The shaping time for $\mathrm{N}_{2}$ was set to $6 \mu$ s and $2 \mu$ s for $\mathrm{CF}_{4}$, corresponding to a different electron drift velocity of about

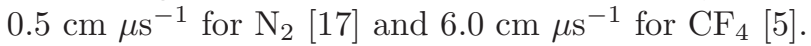

The ranges of the $\alpha$ particles in the different gases have been estimated using Geant4 simulations and found to be around 35 to $42 \mathrm{~mm}$ and 15 to $20 \mathrm{~mm}$ for $\mathrm{N}_{2}$ and $\mathrm{CF}_{4}$ respectively. Due to the uncollimated source, $\alpha$ particles with a large emission angle may not deposit their entire kinetic energy within the active volume in the case of $\mathrm{N}_{2}$.

For $\mathrm{CF}_{4}$, the energy resolutions were extracted from the energy distribution using three Gaussian fits. A background distribution taken as a 9th order polynomial function was added to take care of the above mentioned situation for the $\mathrm{N}_{2}$ energy distribution.

\section{PMT single photoelectron response (SPE)}

Before measuring the scintillation properties of the gases, we performed a calibration of the PMTs in order to obtain the single photoelectron response (SPE) in terms of QDC values. For that, the signals from the PMTs without the source and in ambient air, were acquired in two different time windows. The first time window $\left(\mathrm{QDC}_{1}\right)$ of $35 \mathrm{~ns}$ wide was set around the zero time (-15 ns to $20 \mathrm{~ns}$ ) given by the triggering of the PMT of interest. The trigger level was set to $3 \sigma$ above the electronic noise $(\sim 0.8 \mathrm{mV})$. The second one $\left(\mathrm{QDC}_{2}\right)$ delayed by $45 \mathrm{~ns}$ from 30 to $65 \mathrm{~ns}$, was used to obtain non-correlated signals. The width of the windows has been chosen to exceed more than 10 SPE decay time of the PMT, estimated to be around 3 ns on the signal observed on the oscilloscope. This procedure was done for both PMTs.

Fig. 2 gives the distributions of $\mathrm{QDC}_{1}$ and $\mathrm{QDC}_{2}$ for $\mathrm{PMT}_{1}$. The number of events is normalized by the total number of counts measured during the entire acquisition. $\mathrm{QDC}_{2}$ shows in black the distribution of the pedestal and the noise of the first dynode, described by a Poisson distribution and an exponential decay behaviour [18]. This plot gave the lower limit for the SPE signals at 1500 QDC channels. By fitting the distribution of $\mathrm{QDC}_{1}$ corresponding to 1 photoelectron (phe) induced by thermal agitation on the photocathode with a Gaussian curve (solid red line), we obtained the correspondence of the SPE response as the average value of 2200 QDC channels. Multiple photoelectrons distributions $(\geq 2)$ were not taken into account in the measurement of the single phe.

\section{Scintillation decay times}

The effective decay time of the scintillation processes were measured for up to $100 \mathrm{~ns}$ considering only one decay component.

To measure the decay time spectra, we used a somewhat similar method as given in [19]. One PMT ( $\left.\mathrm{PMT}_{1}\right)$ was used to trig to the single photoelectron level. Its entrance window has then been partially covered in order to reduce the probability to detect two phe from a scintillation event below $0.1 \%$, regarding the approximate scintillation light yield, PMTs detection efficiencies and 


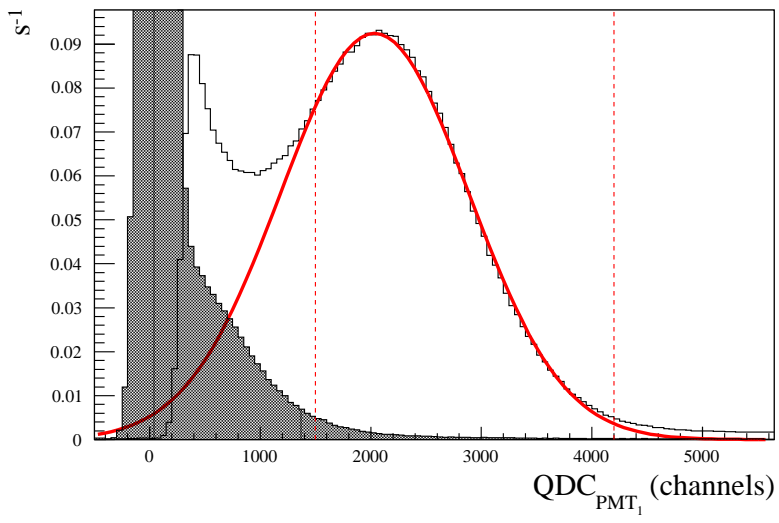

FIG. 2: SPE calibration spectrum for $\mathrm{PMT}_{1}, \mathrm{QDC}_{1}$ is plotted unfilled and $\mathrm{QDC}_{2}$ is black filled. The solid red line correspond to the Gaussian fit of one photoelectron. The vertical dashed lines show the fit intervals.

Poisson statistics. Each PMT output was recorded individually. When put in coincidence with a time window of $100 \mathrm{~ns}$, the second PMT $\left(\mathrm{PMT}_{2}\right)$ detected the scintillation photons corresponding to the energy deposited by the alpha particles. The arrival time of the $\mathrm{PMT}_{1}$ signal ('stop' detector), gave the time difference between the two. The time difference was then plotted to obtain the scintillation time spectra for each gas.

The decay time of the scintillations were extracted using the procedure described in section .

\section{Scintillation emission yield}

The scintillation emission yields of the gases have been measured in terms of photoelectrons converted by the PMT photocathodes. The tri- $\alpha$ source was placed as mentioned above and the gas flowed at 1 atm pressure. The electronic triggers on the PMT signals have been set to $3 \sigma$ above the electronic noise level. The acquisition was done regardless the triggering PMT and data were set in coincidence or in anti-coincidence afterwards in post-processing analysis using their time stamps. The coincidence measurements relate to the scintillation processes issued by $\alpha$ particles from the source while in anticoincidence, background events can be measured.

The integration time window for every triggered event was set to $30 \mathrm{~ns}$ for $\mathrm{N}_{2}$ and $40 \mathrm{~ns}$ for $\mathrm{CF}_{4}$, assuming an approx. $2.5 \mathrm{~ns}$ and $6.3 \mathrm{~ns}$ decay time for $\mathrm{N}_{2}$ and $\mathrm{CF}_{4}$ respectively. The time windows represented then the integration of more than 95\% (3 decay times) of the signals and allowed to avoid after-pulses considerations in the charge integration. Those appeared at $~ 300 \mathrm{~ns}$, $\sim 600$ ns and $\sim 2 \mu \mathrm{s}[20]$ after the main signal in the anticoincidence events.

Fig. 3 shows an example of the energy spectrum mea- sured for $\mathrm{CF}_{4}$ using the tri- $\alpha$ source. The spectra measured shows two components: one in coincidence in the two PMTs showing the primary scintillation induced by $\alpha$ particles, and a second corresponding to after-pulses in the PMT.

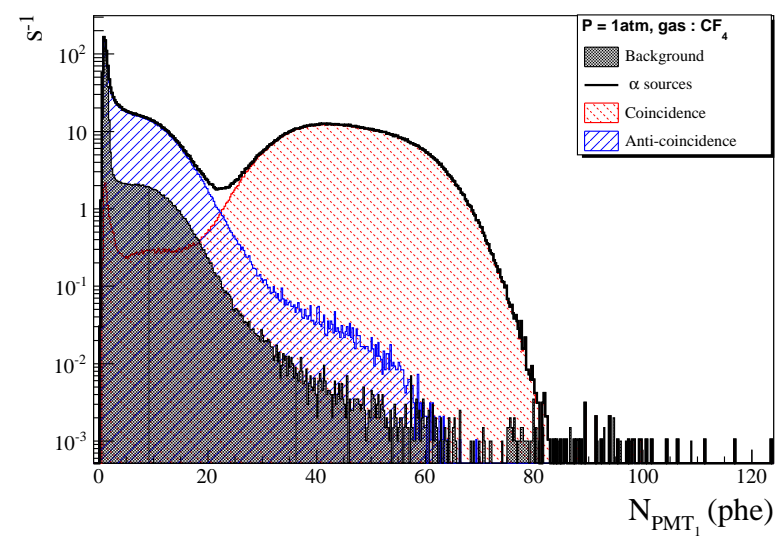

FIG. 3: Distribution of photoelectrons in one PMT induced by $\alpha$ particles (black line histogram) for $\mathrm{CF}_{4}$ at atmospheric pressure, the dashed line filled (red) histogram corresponds to coincidence between the two PMTs and the filled with solid lines (blue) histogram corresponds to anti-coincidence. The dot filled (black) histogram corresponds to background acquisition.

The sum of the two PMTs signals for the coincidence events only were used to extract the scintillation light yields for the two gases. For nitrogen, due to a degraded IC energy resolution and a slower electron drift velocity, no information from the IC was used to help in the selection of the three different $\alpha$ interactions in the gas. For the $\mathrm{CF}_{4}$ gas however, a correlation spectrum between the recorded IC energy and the sum of the two PMTs signals was made. Each $\alpha$ distribution was selected separately and the three spectra of the sum of the PMTs signals were fitted with a Gauss distribution to extract their mean and $\sigma$ values.

The corresponding light yields of each gas were then corrected for the geometrical factors and using the PMTs $\mathrm{SPE}$ responses. The geometrical factors, corresponding to the number of photons incident on the PMTs windows divided by the total number of emitted photons, of $(3.1 \pm 0.2) \%$ and $(3.6 \pm 0.2) \%$ for $\mathrm{N}_{2}$ and $\mathrm{CF}_{4}$ respectively, have been estimated by Monte-Carlo simulations using Geant4-9.5.0 [21]. A detailed description of the simulation is presented in section .

\section{System Coincidence resolving time}

Coincidence resolving time measurements were conducted for the two gases using the two PMTs in coincidence. 
The coincidence time window of $100 \mathrm{~ns}$ was used as well as the appropriate integration time windows for the corresponding gases. Correlation plots were made, after conversion of the QDC channels into SPE, between the number of SPE collected by each PMT. In these distributions, a selection was made accounting for the correlated events. For those events, the time difference of arrival between the two PMTs signals was then plotted and fitted with a Gaussian curve, the coincidence resolving time being the extracted $\sigma$ values.

\section{System simulations}

Monte Carlo simulations were performed for the detection system with two different goals. A first goal, was to accurately extract the scintillation time characteristics of the two gases using phenomenological behavior of the PMT electronic response. The second goal was to build the detection system in Geant4-9.5.0 to be able to perform further simulations where the system intrinsic properties were to be evaluated experimentally. Those two approaches are detailed below.

\section{Scintillation decay time evaluation}

To extract the parameter $\tau_{d}$ of the scintillation process, a Monte-Carlo simulation was used, taking into account the scintillation rise and decay times, the electronic response of the PMTs and triggering system. A number $\mathrm{N}$ of photoelectrons following the time distribution given by $f_{s c}(t)$ in eq. 1 with $\tau_{r}$ the rising time of the system, was generated proportional to the gas emission yield previously measured and the PMTs detection efficiencies. This method was mandatory to match the technique used for measuring the scintillation decay time, where one has to trig on the primary scintillation signal of a PMT while putting in coincidence the triggered signal from one photoelectron of the other PMT. The electrical signals were computed by convolving the photoelectrons temporal distributions with an assumed Gaussian shape of the single photoelectrons from the PMT and the response $h(t)$ of the read-out circuit given in eq. 2 . The circuit was taken as a low-pass filter of the first order with parameters $R$ and $C$, respectively the impedance and the capacitance, chosen to fit its experimental behaviour [22].

$$
\begin{gathered}
f_{s c}(t)=\exp \left(-\frac{t}{\tau_{d}}\right)-\exp \left(-\frac{t}{\tau_{r}}\right), \\
h(t)=\frac{1}{C} \exp \left(-\frac{t}{R C}\right)
\end{gathered}
$$

The signals passed then through dCFDs, to extract for one PMT, the triggering time of the scintillation processes, $t^{s c i}$, and the single phe triggering time, $t^{p h e}$, of the other PMT. Their time difference was then plotted. The parameters $\tau_{r}$ and $\tau_{d}$ were changed until they minimized the $\chi^{2}$ values between the simulated time difference spectra and the experimental ones. Extraction of the rising time was made possible using this method. However, a relevant value would require taking care of all possible parameters (e.g. the range of the $\alpha$ particles, the transit time spread of the PMTs, etc...) which are mainly out the scope of this paper. In consequence, the rising time of the scintillation will not be presented here.

This method was used instead of the traditional exponential fitting procedure in order to take into account for our intrinsic system properties without having to deconvolve them afterwards.

\section{Geant4 system simulations}

The detection system was simulated using Geant49.5.0 to transport the optical photon from the different sources along the $\alpha$ range to the photocathode of the PMTs. The full geometry of our system was implemented including the field shaping wire, the FG and their supports. The tri- $\alpha$ source was modeled by a cylinder at the surface of the aluminum support, the direction emissions were randomized in the hemisphere facing the active volume. Geant 4 comes with a full implementation of electromagnetic processes (using the em_option3 physics package) for taking care of the $\alpha$ particle interactions in the gas. The simulation of the scintillation light emission was as follows. For each $\alpha$ particle energy deposition, the simulation generated a number of photons proportional to the gas emission yields, emitted isotropically at the wavelength at maximum emission, with two components in the case of $\mathrm{CF}_{4}$. All the system surfaces optical behaviors were taken into account using the unified model included in Geant4. The photon passing through the PMTs entrance windows were converted in post-analysis treatment into photoelectrons using the corresponding measured quantum efficiencies found in [15]. A simulated PMT signal and read-out electronic was then generated using the same procedure as described in section .

The resulting calculations have been compared with the experimental data concerning the coincidence resolving times between the two PMTs.

\section{RESULTS AND DISCUSSION}

\section{IC energy resolution}

Fig. 4 shows the energy distributions of the tri- $\alpha$ source for the two different gases. The energy resolution of each 


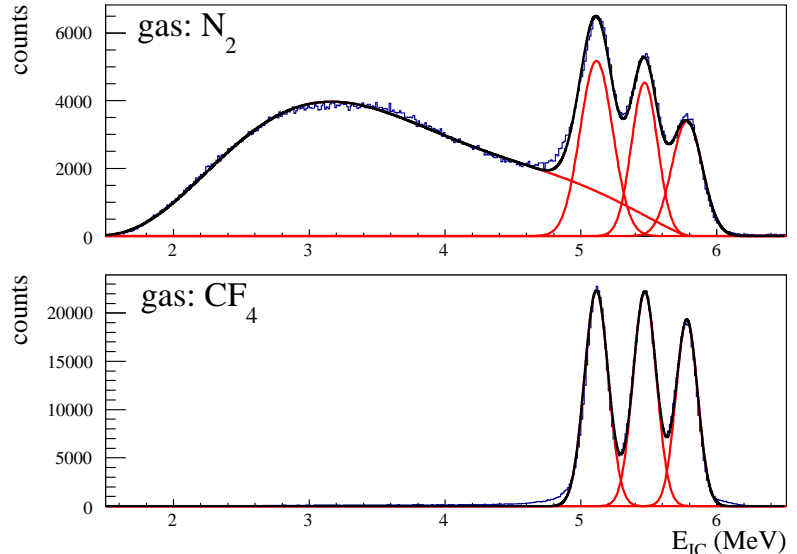

FIG. 4: Energy spectra obtained from the ionisation chamber for the two gases with the tri- $\alpha$ source.

gas is extracted by fitting each peak with a Gauss distribution plus a background distribution in the case of $\mathrm{N}_{2}$. Table I presents the different resolutions obtained for our energy measurements.

The energy resolution obtained with $\mathrm{CF}_{4}$ is slightly better than for $\mathrm{N}_{2}$ with a minimum value of $3.2 \%$ measured on the $5.8 \mathrm{MeV}{ }^{244} \mathrm{Cm} \alpha$ emission. This difference can be explained by a Fano factor smaller in the case of $\mathrm{CF}_{4}$ compared to $\mathrm{N}_{2}$ with respectively $\mathrm{F} \simeq 0.2[23]$ and $\mathrm{F} \simeq 0.28$ [24]. Nevertheless, the energy resolutions allow the three different peaks to be clearly visible in both gases.

TABLE I: Energy resolution of the ionization chamber referred as the FWHM in $\mathrm{keV}$ of each $\alpha$ peak for the different gases. In parentheses, the relative energy resolution in $\%$.

\begin{tabular}{|c|c|c|}
\hline $\mathrm{E}_{\alpha}(\mathrm{MeV})$ & $\mathrm{N}_{2}$ & $\mathrm{CF}_{4}$ \\
\hline 5.14 & 336. $(6.5 \%)$ & 204. (4.0\%) \\
\hline 5.44 & 270. (5.0\%) & 200. $(3.7 \%)$ \\
\hline 5.80 & 250. (4.3\%) & 188. $(3.2 \%)$ \\
\hline
\end{tabular}

\section{Scintillation decay times and emission yields}

Fig. 5 shows the measured (black solid lines) and simulated (red dashed lines) scintillation time spectra obtained for $\mathrm{N}_{2}$ and $\mathrm{CF}_{4}$ gases.

Decay times are respectively extracted at $(2.5 \pm 0.5) \mathrm{ns}$ and $(6.2 \pm 0.8)$ ns for $\mathrm{N}_{2}$ and $\mathrm{CF}_{4}$ with $\chi^{2} / n d f$ values of 1.45 and 1.1. Those results are in good agreement with previously published ones $[9,10]$ and show that $\mathrm{N}_{2}$ is 2.5 times faster than $\mathrm{CF}_{4}$.

Fig. 6 shows the sum of the number of photoelectrons collected by each PMT for the two gases. In the case of $\mathrm{N}_{2}$, no selections were made on the IC energy to select

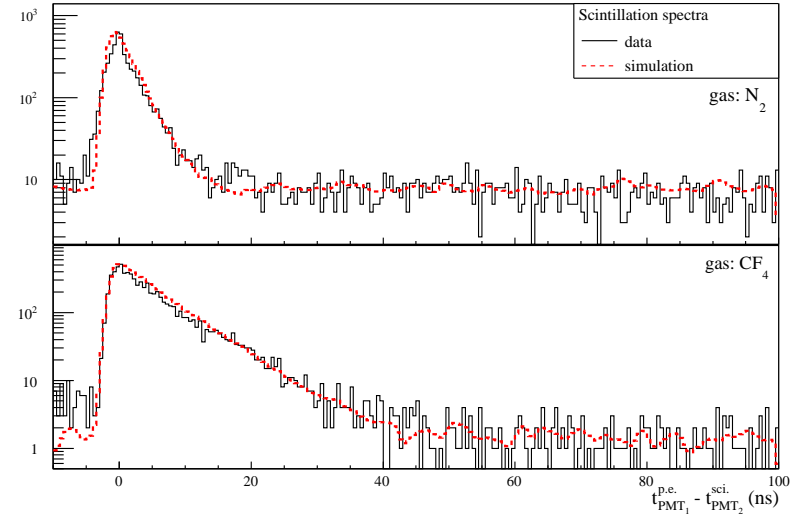

FIG. 5: Experimental time scintillation spectra for $\mathrm{N}_{2}$ and $\mathrm{CF}_{4}$ gases and the simulation used to extract the timing properties (red dashed lines).

individual $\alpha$ emitter. The number of photoelectrons corrected from the detection efficiencies for $\mathrm{N}_{2}$, corresponds thereby to a mean value of 24 phe $\mathrm{MeV}^{-1}$ with a standard deviation of 10 phe $\mathrm{MeV}^{-1}$.

In the case of $\mathrm{CF}_{4}$, the selection of each $\alpha$ emitter using the IC energy was made and fitted using a Gauss distribution (Fig. 6(b)). The mean phe values are $41 \pm 5,44 \pm 5$ and $46 \pm 5$ for $5.14,5.44$ and $5.8 \mathrm{MeV}$ $\alpha$ energies respectively, corresponding to a mean number of photoelectrons corrected for the detection efficiencies of $(225 \pm 28)$ phe $\mathrm{MeV}^{-1}$. Using the measured quantum efficiencies given in [15] for our PMTs of $25 \%$ for $\mathrm{N}_{2}$ and $19 \%$ for a composition of the two components of $\mathrm{CF}_{4}$ (evaluated at approx. 2/3 and $1 / 3$ for the 300 and $630 \mathrm{~nm}$ emissions at a 1 atm pressure [7]), photon yields of $(96 \pm 40) \mathrm{ph} \mathrm{MeV}^{-1}$ for nitrogen and $(1184 \pm 47) \mathrm{ph} \mathrm{MeV}^{-1}$ for tetrafluoromethane are extrapolated. Those results, intended for comparison, are found to be in good agreement with previously published materials $[6,11]$. However, the $\mathrm{CF}_{4}$ scintillation light yield differs from the results of [7] by a factor 2. As mentioned by this study, the contaminants in the gas are of great importance on the number of photons emitted, which we also observed when comparing measurements from a different gas provider (not shown).

\section{Coincidence resolving time}

Fig. $7(\mathrm{a}, \mathrm{c})$ show the correlation distribution between the number of phe in each PMT for $\mathrm{N}_{2}$ and $\mathrm{CF}_{4}$ respectively, at $1 \mathrm{~atm}$ pressure. The red dashed lines represent the selections used to make the time coincidence distributions in Fig. 7(b,d).

The CRT measured values are $\sigma=1.4$ ns for $\mathrm{N}_{2}$ and $\sigma=0.34 \mathrm{~ns}$ for $\mathrm{CF}_{4}$. Despite of a decay time for $\mathrm{CF}_{4}$ two times slower than the one for $\mathrm{N}_{2}$, the CRT for the 

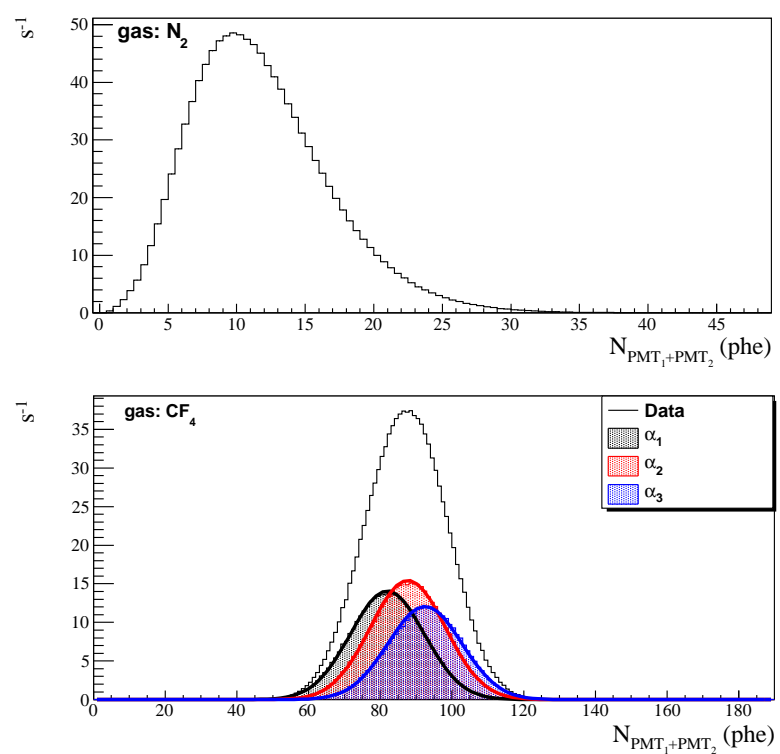

FIG. 6: (top) Sum of the photoelectrons seen by the PMTs for $\mathrm{N}_{2}$. (bottom) Sum of the photoelectrons seen by the PMTs for each $\alpha$ energy selected by the IC and fitted by a Gaussian distribution for $\mathrm{CF}_{4}$.

$\mathrm{CF}_{4}$ has been improved by a factor 4 . This can be attributed to a higher emission yield of the scintillation process, considering that the CRT evaluation follows a $\left(\mathrm{N}_{\text {phe }}\right)^{-1 / 2}$ trend [25] when the photodetectors and the trigger thresholds were the same for both gases.

\section{System simulations}

The decay times and the scintillation emission yields given in section were used as input parameters for the simulation of the detection system.

The time coincidence distributions are compared to the experimental ones in Fig. 8. Considering the large approximations that were made, for instance in the conversion of the scintillation emission yields, the simulation of the PMTs electronic responses and the trigger system coupled with the Geant4 simulation allowed us to reproduce the coincidence resolving times for both gases with a correlation factor of 0.995 for both gases. Therefore, we estimated that further simulations of the system can be used using this procedure to evaluate the most appropriate conditions for the detection of heavier fission fragments.

\section{CONCLUSION}

Concerning the FALSTAFF project for the detection of fission fragments, the use of a scintillating ionisation
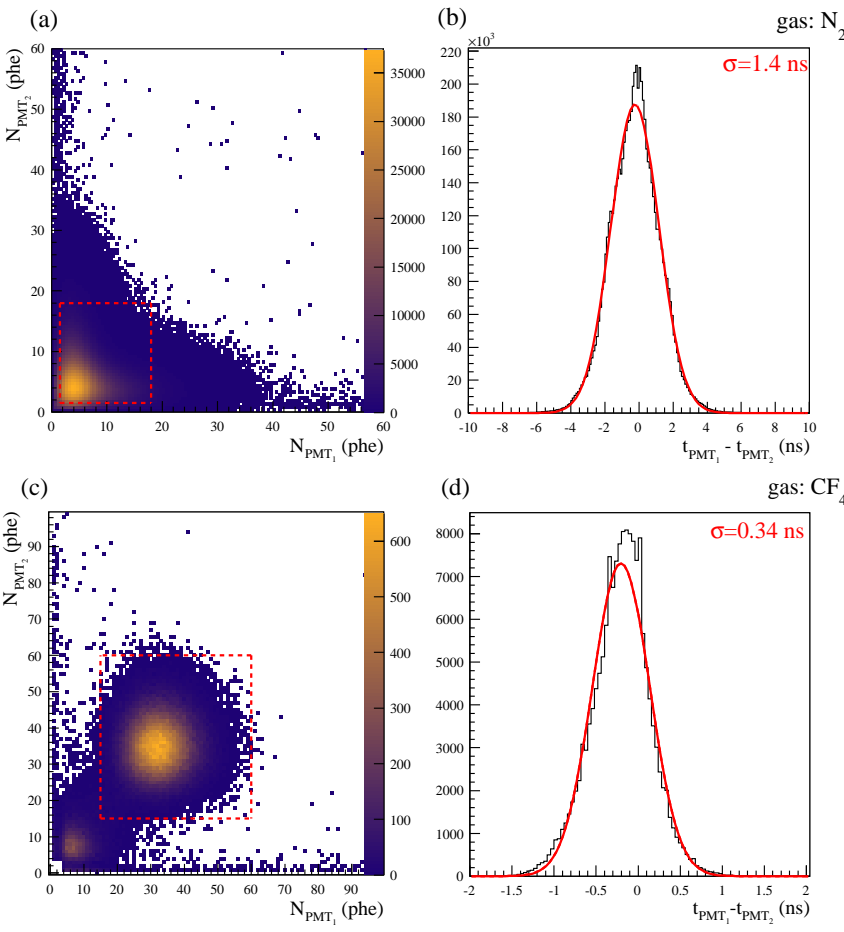

FIG. 7: (a) Correlation plot and (b) time coincidence distribution for $\mathrm{N}_{2}$, and (c), correlation plot and (d) coincidence time spectrum for $\mathrm{CF}_{4}$. The red dashed lines indicate the selections made for building the coincidence time spectra, the red solid lines represent the Gaussian fits applied to extract the coincidence time resolutions.
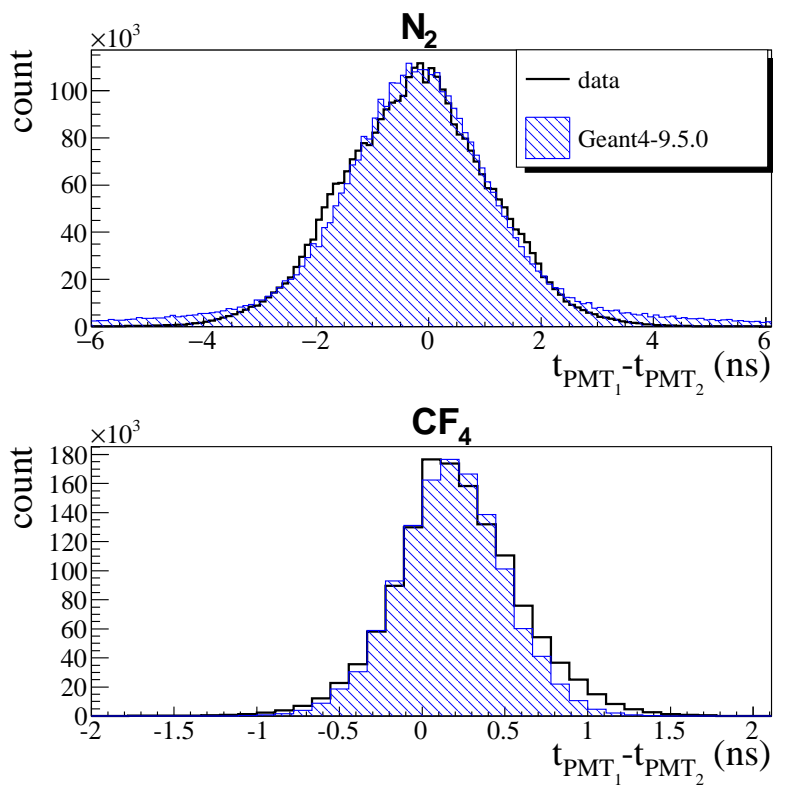

FIG. 8: Time coincidence distributions measured (solid black line) and simulated (blue strips filled) between the two PMTs in (a) $N_{2}$ and (b) $\mathrm{CF}_{4}$. 
chamber could not achieve a better ToF resolution for the system than the CRT of the PMTs, even though it would be expected around 150 ps for higher deposited energies. The typical time resolution achieved by a Secondary Electron Detector primarily considered for this project, can in principle reach sigma values better than $130 \mathrm{ps}$ and a $1.5 \mathrm{~mm}$ spatial resolution [26]. As a result, SEDs seem more suited than a scintillating ionization chamber to fulfill the requirements of this project.

However, the scintillating ionization chamber can be used as an active gaseous target for cross section measurements of interest for nuclear reactions induced by neutrons, envisioned at NFS [27]. This kind of experiment would require further studies on the scintillation properties of specific gaseous mixtures, completing some of the existing data $[11,28]$. These studies are in progress and results are expected in a close future.

\section{Acknowledgements}

This work was partly supported by the French NEEDS programs of the CNRS. The authors would like to thank the technical staff of the LPC Caen for the realization of the detection system.

[1] F. Tovesson, C. Arnold, R. Blakeley, A. Hecht, A. Laptev, D. Mader, K. Meierbachtol, L. Snyder, and M. White. SPIDER: A new instrument for fission fragment research at the los alamos neutron science center. EPJ Web of Conferences, 62:05002, 2013.

[2] D. Doré, F. Farget, F.-R. Lecolley, X. Ledoux, G. Lehaut, T. Materna, J. Pancin, S. Panebianco, and the FALSTAFF and NFS Collaborations. Fission fragment characterization with FALSTAFF at NFS. EPJ Web of Conferences, 42:01001, 2013.

[3] T. Shima, K. Watanabe, T. Irie, H. Sato, and Y. Nagai. A gas scintillation drift chamber for the $14 \mathrm{~N}(\mathrm{n}, \mathrm{p}) 14 \mathrm{C}$ measurement. Nuclear Instruments and Methods in Physics Research Section A: Accelerators, Spectrometers, Detectors and Associated Equipment, 356(23):347 - 355, 1995.

[4] I.P. Bondarenko, V.A. Khryachkov, T.A. Ivanova, B.D. Kuzminov, N.N. Semenova, and A.I. Sergachev. Experimental investigation of the $19 \mathrm{f}(\mathrm{n}) ,16 \mathrm{n}$ reaction excitation function in the neutron energy range of 4 to $7.35 \mathrm{mev}$. Physics of Particles and Nuclei Letters, 10(4):349-352, 2013.

[5] J. Va'vra, P. Coyle, J. Kadyk, and J. Wise. Measurement of electron drift parameters for helium and CF4-based gases. Nuclear Instruments and Methods in Physics Research Section A: Accelerators, Spectrometers, Detectors and Associated Equipment, 324(12):113 - 126, 1993.

[6] A. Pansky, A. Breskin, A. Buzulutskov, R. Chechik, V. Elkind, and J. Va'vra. The scintillation of CF4 and its relevance to detection science. Nuclear Instruments and Methods in Physics Research Section A: Acceler- ators, Spectrometers, Detectors and Associated Equipment, 354(23):262 - 269, 1995.

[7] A. Morozov et al. Photon yield for ultraviolet and visible emission from cf4 excited with alpha-particles. Nuclear Instruments and Methods in Physics Research Section B: Beam Interactions with Materials and Atoms, 268:1456 $1459,2010$.

[8] A. Morozov et al. Effect of the electric field on the primary scintillation from cf4. Nuclear Instruments and Methods in Physics Research Section A: Accelerators, Spectrometers, Detectors and Associated Equipment, 628:360 - 363, 2011.

[9] L.M.S. Margato, A. Morozov, L. Pereira, M.M.F.R. Fraga, and F.A.F. Fraga. Effect of the gas contamination on CF4 primary and secondary scintillation. $\mathrm{Nu}$ clear Instruments and Methods in Physics Research Section A: Accelerators, Spectrometers, Detectors and Associated Equipment, 695:425 - 428, 2012.

[10] J. Liu, X. Ouyang, Chen L., X. Zhang, J. Liu, Z. Zhang, and J. Ruan. Primary scintillation characteristics of $\mathrm{Ar}+\mathrm{CF} 4$ gas mixtures excited by proton and alpha particles. Nuclear Instruments and Methods in Physics Research Section A: Accelerators, Spectrometers, Detectors and Associated Equipment, 694:157-161, 2012.

[11] H. Morii, K. Mizouchi, T. Nomura, N. Sasao, T. Sumida, M. Kobayashi, Y. Murayama, and R. Takashima. Quenching effects in nitrogen gas scintillation. Nuclear Instruments and Methods in Physics Research Section A: Accelerators, Spectrometers, Detectors and Associated Equipment, 526(3):399 - 408, 2004.

[12] F. Hecht. New development in freefem ++ . J. Numer. Math., 20(3-4):251-265, 2012.

[13] A. Gook, F.-J. Hambsch, A. Oberstedt, and S. Oberstedt. Application of the shockleyramo theorem on the grid inefficiency of frisch grid ionization chambers. $\mathrm{Nu}$ clear Instruments and Methods in Physics Research Section A: Accelerators, Spectrometers, Detectors and Associated Equipment, 664(1):289 - 293, 2012.

[14] J.B. Birks. The Theory and Practice of Scintillation Counting. MacMillan New York, 1964.

[15] P. Dorenbos, J.T.M. de Haas, R. Visser, C.W.E. van Eijk, and R.W. Hollander. Quantum efficiencies of several vuv-sensitive photomultiplier tubes. Nuclear Instruments and Methods in Physics Research Section A: Accelerators, Spectrometers, Detectors and Associated Equipment, 325(12):367-369, 1993.

[16] X. Fléchard, E. Liénard, O. Naviliat-Cuncic, D. Rodriguez, M. Alvarez, G. Ban, B. Carniol, D. Etasse, J. Fontbonne, A. Lallena, and J. Praena. Measurement of the ${ }^{8}$ Li half-life. Phys. Rev. C, 82:027309, 2010.

[17] R. Veenhof. http://garfield.web.cern.ch/garfield/. CERN.

[18] E.H. Bellamy, G. Bellettini, J. Budagov, F. Cervelli, I. Chirikov-Zorin, M. Incagli, D. Lucchesi, C. Pagliarone, S. Tokar, and F. Zetti. Absolute calibration and monitoring of a spectrometric channel using a photomultiplier. Nuclear Instruments and Methods in Physics Research Section A: Accelerators, Spectrometers, Detectors and Associated Equipment, 339(3):468 - 476, 1994.

[19] L. M. Bollinger and G. E. Thomas. Measurement of the time dependence of scintillation intensity by a delayedcoincidence method. Review of Scientific Instruments, 32(9):1044-1050, 1961.

[20] U. Akgun, A. S. Ayan, G. Aydin, F. Duru, J. Olson, and 
Y. Onel. Afterpulse timing and rate investigation of three different hamamatsu photomultiplier tubes. Journal of Instrumentation, 3(01):T01001, 2008.

[21] S. Agostinelli et al. Geant4 - a simulation toolkit. $\mathrm{Nu}$ clear Instruments and Methods in Physics Research Section A: Accelerators, Spectrometers, Detectors and Associated Equipment, 506(3):250 - 303, 2003.

[22] S. Liu, H. Li, Y. Zhang, R.A. Ramirez, H. Baghaei, S. An, C. Wang, J. Liu, and W.-H. Wong. Monte carlo simulation study on the time resolution of a PMT-quadrantsharing LSO detector block for time-of-flight PET. $\mathrm{Nu}$ clear Science, IEEE Transactions on, 56(5):2614-2620, 2009.

[23] W.S. Anderson, J.C. Armitage, E. Dunn, J.G. Heinrich, C. Lu, K.T. McDonald, J. Weckel, and Y. Zhu. Electron attachment, effective ionization coefficient, and electron drift velocity for $\{\mathrm{CF} 4\}$ gas mixtures. Nuclear Instruments and Methods in Physics Research Section A: Accelerators, Spectrometers, Detectors and Associated Equipment, 323(12):273 - 279, 1992.

[24] G.D. Alkhazov, A.P. Komar, and A.A. Vorob'ev. Ioniza- tion fluctuations and resolution of ionization chambers and semiconductor detectors. Nuclear Instruments and Methods, 48(1):1 - 12, 1967.

[25] L. G. Hyman, R. M. Schwarcz, and R. A. Schluter. Study of high speed photomultiplier systems. Review of Scientific Instruments, 35(3):393-406, 1964.

[26] S. Panebianco, D. Doré, F. Farget, F.-R. Lecolley, G. Lehaut, Th. Materna, J. Pancin, and Th. Papaevangelou. FALSTAFF : a novel apparatus for fission fragment characterization. EPJ Web of Conferences, 69:00021, 2014.

[27] X. Ledoux et al. The neutrons for science facility at spiral-2. Nuclear Data Sheets, 119:353 - 356, 2014.

[28] W. Lorenzon, O. Husser, and T. Plettner. Gas scintillation in He N2 CH4 and He N2 mixtures. Nuclear Instruments and Methods in Physics Research Section A: Accelerators, Spectrometers, Detectors and Associated Equipment, 342(23):516 - 520, 1994.

[29] Neutron For Science at SPIRAL2 in GANIL 